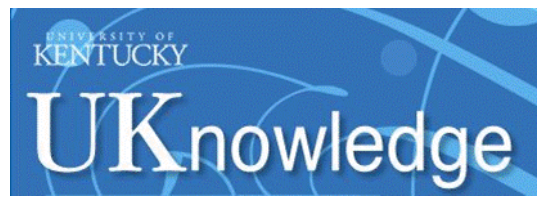

University of Kentucky

UKnowledge

\title{
An Aggregated and Equivalent Home Model for Power System Studies with Examples of Building Insulation and HVAC Control Improvements
}

\author{
Huangjie Gong \\ University of Kentucky, huangjie.gong@uky.edu \\ Evan S. Jones \\ University of Kentucky, SEvanJones@uky.edu \\ Dan M. Ionel \\ University of Kentucky, dan.ionel@uky.edu
}

Follow this and additional works at: https://uknowledge.uky.edu/peik_facpub

Part of the Power and Energy Commons

Right click to open a feedback form in a new tab to let us know how this document benefits you.

\section{Repository Citation}

Gong, Huangjie; Jones, Evan S.; and Ionel, Dan M., "An Aggregated and Equivalent Home Model for Power System Studies with Examples of Building Insulation and HVAC Control Improvements" (2020). Power and Energy Institute of Kentucky Faculty Publications. 64.

https://uknowledge.uky.edu/peik_facpub/64

This Conference Proceeding is brought to you for free and open access by the Power and Energy Institute of Kentucky at UKnowledge. It has been accepted for inclusion in Power and Energy Institute of Kentucky Faculty Publications by an authorized administrator of UKnowledge. For more information, please contact UKnowledge@lsv.uky.edu. 


\title{
An Aggregated and Equivalent Home Model for Power System Studies with Examples of Building Insulation and HVAC Control Improvements
}

\author{
Digital Object Identifier (DOI) \\ https://doi.org/10.1109/PESGM41954.2020.9281438
}

\section{Notes/Citation Information}

Published in 2020 IEEE Power \& Energy Society General Meeting (PESGM).

(C) 2020 IEEE Copyright Notice. "Personal use of this material is permitted. Permission from IEEE must be obtained for all other uses, in any current or future media, including reprinting/republishing this material for advertising or promotional purposes, creating new collective works, for resale or redistribution to servers or lists, or reuse of any copyrighted component of this work in other works."

The document available for download is the authors' manuscript version accepted for publication. The final published version is copyrighted by IEEE and is available as: H. Gong, E. S. Jones, and D. M. Ionel, "An Aggregated and Equivalent Home Model for Power System Studies with Examples of Building Insulation and HVAC Control Improvements," Rec. 2020 IEEE Power \& Energy Society General Meeting (PESGM), Montreal, Canada, Aug 2020, pp. 1-4, doi: 10.1109/PESGM41954.2020.9281438 


\title{
An Aggregated and Equivalent Home Model for Power System Studies with Examples of Building Insulation and HVAC Control Improvements
}

\author{
Huangjie Gong, Evan S. Jones, and Dan M. Ionel \\ SPARK Laboratory, Department of Electrical and Computer Engineering, University of Kentucky, Lexington, KY, US \\ huangjie.gong@ieee.org, SEvanJones@uky.edu, dan.ionel@ieee.org
}

\begin{abstract}
Introducing new technologies into existing residences provides opportunities for the utility to enhance a community's power system performance. To validate the benefits of such technologies, an energy model is required in which their integration with a community's power system may be simulated. It can be difficult or impossible to properly model a power system without sufficient sample data. This paper proposes a method that uses Gaussian kernel density estimation (GKDE) to calculate the aggregated net power flow of a community's distribution system with only limited sample points at each time step. Example case studies that confirm the usefulness of the GKDE method are presented in which the power system benefits of improved insulation and heating, ventilation, and air conditioning (HVAC) system control are analyzed. This analysis was performed using an EnergyPlus (EP) house model that was created and calibrated based upon an individual house. This power representation of the individual house was determined by accurately estimating the aggregated net power flow of a community in Glasgow, KY with GKDE. Simulation results based on the equivalent house energy model show that improved insulation reduces the energy consumption and the peak power at the power system level. Simulation results also show that the HVAC control reduces the peak power for the entire community.
\end{abstract}

Keywords-Gaussian Kernel Density Estimation (GKDE); Aggregated Net Power Flow; Distribution Power System; EnergyPlus; HVAC Control; Home Energy Management (HEM)

\section{INTRODUCTION}

The residential sector in the US consumed $3.8 \times 10^{6}$ GWh of energy in 2018 [1]. Because of this large amount of energy consumption, new smart home technologies are attracting more interest. Such technologies have great potential in achieving highly efficient operation at the distribution power system level. The development of the Internet-of-things (IoT) has also promoted the adoption of these new technologies by enabling the coordination of individual houses to collectively realize specific objectives. The aggregated effect of controlling individual houses has become ever more important as more residences are able to respond to external signals.

By considering existing residences as a large thermal mass, HVAC systems can be updated to enable home energy management (HEM). According to a 2015 survey by the US Energy Information Administration (EIA), heating, ventilation, and air conditioning (HVAC) systems consume $32 \%$ of total residential electricity [2]. The potential benefits of HVAC control in individual houses have drawn the attention of previous research on ancillary services for the grid such as frequency regulation and demand response [3], [4].

HVAC systems in a power system may be regarded as a fleet and controlled collectively. House owners have indoor temperature preferences, based on which the corresponding HVAC has its cycling period of on and off states [5]. The control for HVAC systems with variable fans can be realized by changing thermostat setting points [6].

The HVAC systems in individual houses differ due to various parameters including insulation, efficiency, and customer preference, even when they are exposed to the same external environment [7]. Modeling the distribution power system and representing the various behaviors of individual HVAC systems has been challenging for previous research work. Gaussian kernel density estimation (GKDE) provides alternative ways of estimating the aggregated load for the entire power system based on limited sample points. Research exists in which a model for short-term load forecasts was developed using GKDE, by which the probability density function (PDF) of the net power flow was obtained [8].

In this paper, the net power flow of a residential community in Glasgow, KY was estimated based on limited sample points using GKDE. The estimated net power flow was determined to have great agreement with the experimental data. An EnergyPlus (EP) house model was created based on the GKDE results to represent an equivalent individual house from within the Glasgow community. The EP model was then used to test the effects of insulation improvement and HVAC control when implemented in individual houses. Cases were studied that verified the benefits of each method at the power system level.

\section{Gaussian Kernel Density Estimation}

Let the data set $X=\left\{x_{1}, x_{2}, \ldots, x_{N}\right\}$ with $N$ being the samples from the distribution of unknown probability density $f$. The GKDE is used to estimate the shape of the distribution, described as,

$$
\widehat{f_{G}}(x ; h)=\frac{1}{N} \sum_{i=1}^{N} K\left(x-x_{i} ; h\right),
$$




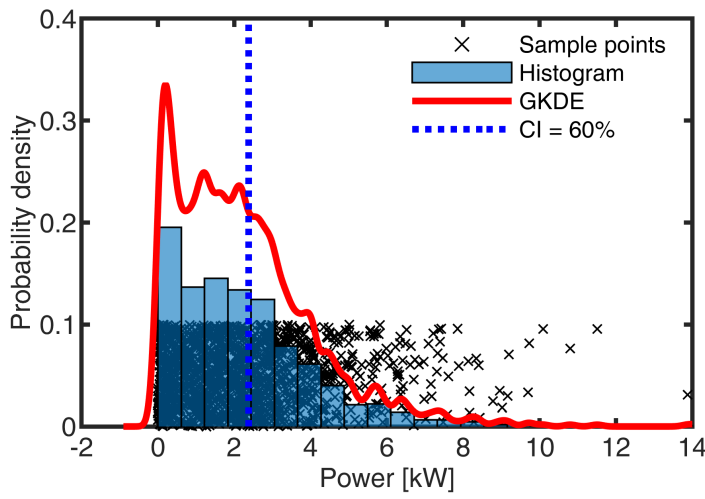

Figure 1. Scattered sampled points at 12:30 with the corresponding histogram and GKDE curve. The bandwidth was set to 0.15. Confidence interval (CI) was set to $60 \%$ on the high power side.

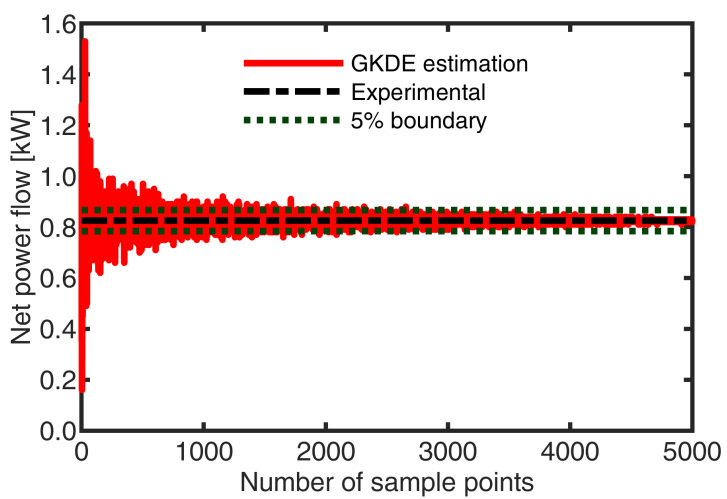

Figure 2. The estimated net power flow converges to the experimental value as more sample points are taken. In the study, 1,500 sample points were taken to estimate the Gaussian kernel density.

where $f_{G}(x ; h)$ is the estimated GKDE; $K$, the kernel function, which is the Gaussian probability density function (PDF) defined as,

$$
K\left(x-x_{i} ; h\right)=\frac{1}{\sqrt{2 \pi h}} e^{\frac{\left(x-x_{i}\right)^{2}}{2 h}},
$$

where $\mathrm{h}$ is the bandwidth. The bandwidth should be large enough to reduce the variance of the PDF and small enough to mitigate the bias. Silverman's rule, which assumes that the true density is the Gaussian distribution, helps to pick the proper bandwidth but might not always work for the practical cases.

In this study, the bandwidth was set to 0.15 after testing the experimental data. An example at one time point including scattered sample points as well as a corresponding histogram and GKDE is shown (Fig. 1). Compared with the histogram, the GKDE is endowed with smoothness and continuity.

The expected value of the GKDE is defined as follows,

$$
E[x]=\int x \widehat{f_{G}}(x ; h),
$$

where $\widehat{f_{G}}(x ; h)$ is the GKDE curve defined in (1). The expected value was compared with the mean value of the

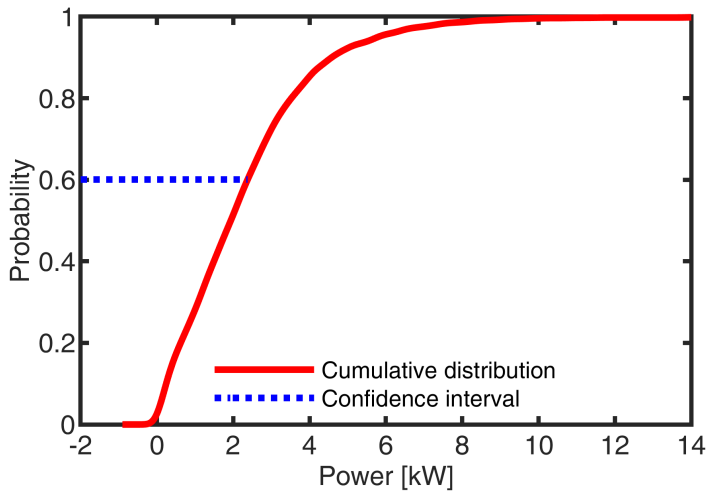

Figure 3. The CDF curve of the GKDE at 12:30. Confidence interval (CI) was set to $60 \%$ on the high power side.

experimental data (Fig. 2). There is trade-off for the number of sample points. Although a large number could avoid randomness and represent the real distribution at maximum, a small number is more desirable in practice considering the cost and accessibility of the data. In this paper, the number of sample points was set to 1,500 as the expected value converges to the experimental data within $5 \%$ of variance.

\section{Modeling And VAlidation for the AgGRegated NET POWER FLOW AND REPRESENTATIVE HOUSE}

The community of Glasgow, KY has a residential distribution power system that includes more than 5,000 houses [6]. Modeling for the aggregated net power flow of the entire power system enables the estimation and analysis of the influences future technologies may have on smart homes. In the study, the net power flow of all 5,000 residences on 7/14/2017 is available, with a time step of 15 -minutes.

In a previous study, the power system is modeled using a modified IEEE 13-feeder test case with all of the residential data fed into the simulation [6]. The GKDE provides a method of estimating the aggregated net power flow of the entire distribution power system instead of directly solving for the power flow. Another advantage in adopting the GKDE is the reduction in necessary sample points. Only 1,500 sample points out of more than 5,000 are needed for an accurate estimation of the entire system.

The sample data was a collection of net power flow values from 1,500 randomly selected houses. At each time point, the Gaussian kernel estimator calculated the PDF of the GKDE using the sample data. The example PDF and cumulative distribution function (CDF) at 12:30 are shown (Fig. 1 and Fig. 3). A confidence interval of $60 \%$ was drawn in both PDF and $\mathrm{CDF}$ on the high power side.

The estimated aggregated net power flow for the entire power system at time $t$ was calculated as follows,

$$
\widehat{P}_{t}=E[x] * M,
$$

where $M$ is the total house number and $E[x]$ is the expected value of the GKDE defined in (3). 


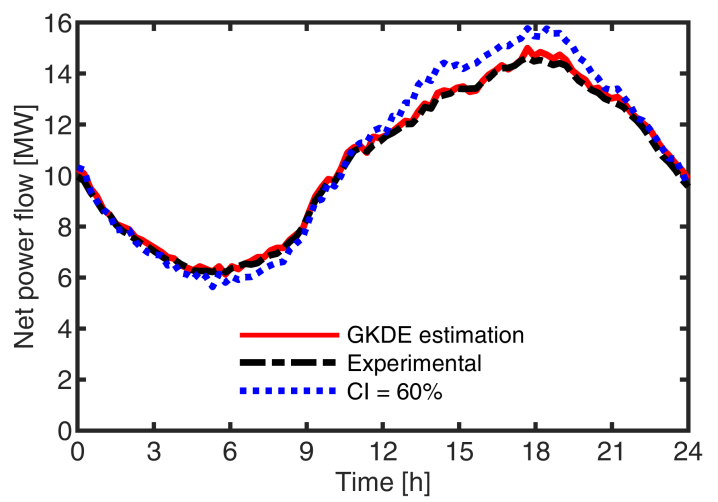

Figure 4. The estimated aggregated net power flow of the entire distribution system on a representative summer day. The GKDE estimation is satisfactory compared with the experimental data. The net power flow was estimated at each sampled time point.

The same procedure was repeated for all 96 time points on $7 / 14 / 2017$. The results of estimating the representative summer day is shown (Fig. 4). The estimated net power flow curve had good agreement with the experimental data, which was the aggregated net power flow of the entire distribution power system. In cases where no aggregated data is available for direct comparison, the proposed GKDE method can provide satisfactory results when the confidence interval is set to $60 \%$ on the high power side.

The estimated aggregated net power flow was then scaled down to a single house that was considered as an accurate estimation of an individual house from the distribution power system. An EP model for the equivalent house was produced and based on the scaled down estimation. The model was then calibrated until its net power flow met satisfactory agreement with the GKDE result (Fig. 5). The EP model for the equivalent house provided the testbed for smart homes, based on which the influences of advanced technologies on the distribution power system can be analyzed. The improved insulation and HVAC control were applied to the equivalent EP model, and its results were scaled up to represent the entire power system. The results and analysis were given in the following section.

\section{CASE Studies For IMPRoved InSUlation AND HVAC CONTROL}

Case studies were carried out to determine the influences that improved insulation and HVAC control have on the residential distribution power system when implemented in the community's houses. In each study, cases representing different technology penetrations were analyzed based on the EP simulation results. A typical summer day was selected for the case studies considering that almost all of the air conditioning was powered by electricity. The weather data used in the EP simulation is the historic data of Glasgow, KY in 2017. The simulation results were recorded every 15 minutes just as the experimental data was.

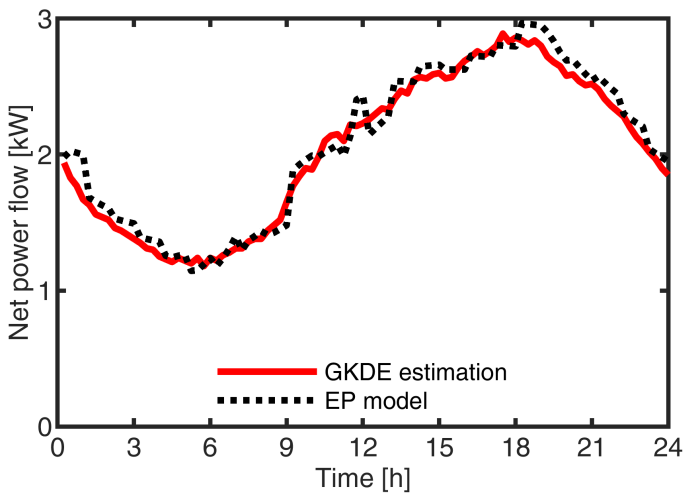

Figure 5. The net power flow of the equivalent house. The EnergyPlus (EP) model is calibrated to represent the equivalent house of the entire distribution power system.

In the first study, the effects of the improved insulation were tested. The equivalent EP model was modified to have improved insulation. In the simulation, the thermostat setting points for both equivalent EP model and EP model with improved insulation were set to $75^{\circ} \mathrm{F}$ constantly (Fig. 6). It is worth noticing that around 15:00 to 18:00, the HVAC had unmet hours due to the high dry bulb temperature (DBT).

Case studies were carried out as baseline, where no improved insulation was implemented; Case 2, where $50 \%$ of the houses had the improved insulation; Case 3, where all the houses were with the improved insulation (Fig. 7). As more houses implemented the improved insulation, the overall energy consumption and peak power demand reduced as expected.

The second study verified HVAC control as an effective scheme for reducing the aggregated peak power. Three cases were carried out with each having different penetrations of HVAC being controlled. Case baseline represents the traditional case where no HVAC was controlled; In Case 2, 50\% of the houses have their HVAC systems being controlled; Case 3 has $100 \%$ of the houses with HVAC control.

The HVAC control was realized by changing the thermostat setting point. To implement HVAC control, the thermostat setting point was changed from $75^{\circ} \mathrm{F}$ to $78^{\circ} \mathrm{F}$ at $16: 00$ when the peak power occurred (Fig. 8). The thermostat setting point was gradually reset to $75^{\circ} \mathrm{F}$ at 21:00 after the peak power time.

Under the HVAC control, the peak power of the distribution system was reduced between 16:00 and 21:00 (Fig. 9). As more houses in the simulation, $50 \%$ for Case 2 and $100 \%$ for Case 3, had their HVAC systems being controlled, peak power reduced within the associated time frame. Changing the thermostat setting point to a lower value at 21:00 caused the peak power for both Case 2 and Case 3. In order to avoid a consequentially high peak, the thermostat setting point was resumed to $75^{\circ} \mathrm{F}$ gradually.

\section{CONClusion}

In this paper, GKDE was proven to be a useful tool for accurately estimating the aggregated net power flow of a 


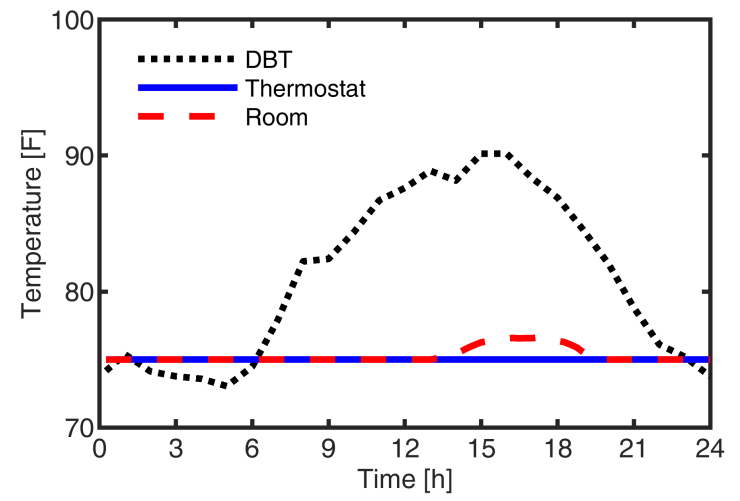

Figure 6. Dry bulb temperature (DBT), thermostat setting point, and room temperature for the equivalent house. The thermostat was set to $75^{\circ} \mathrm{F}$ all day. The high DBT in the afternoon caused unmet hours for the HVAC system and peak power flow for the entire distribution system.

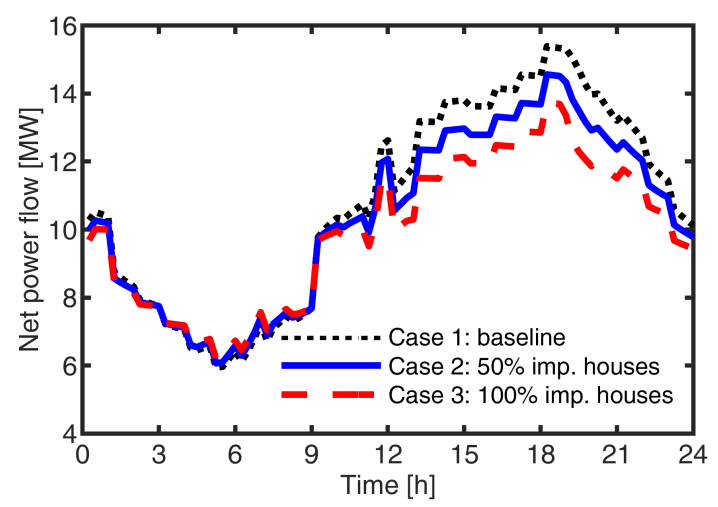

Figure 7. Simulation results for case studies of improved insulation. Energy consumption and peak power reduced as penetration of improved insulation increased.

community distribution system using limited data. An EP house model was developed and calibrated based on the GKDE results and used to represent the equivalent home of the entire community. The EP model was then used as a testbed to verify the benefits of new technologies for the community.

Case studies were completed to serve as examples of the GKDE method's utility, which included the analysis of insulation and HVAC control improvement effects on a community's power system. Simulation results show that improved insulation of individual houses could reduce both peak power and energy usage at the power system level. It was also found that HVAC control in residences can reduce peak power at the power system level.

\section{ACKNOWLEDGMEnT}

The support of University of Kentucky, the L. Stanley Pigman endowment, and of the SPARK program and the Power and Energy Institute of Kentucky (PEIK) is gratefully acknowledged.

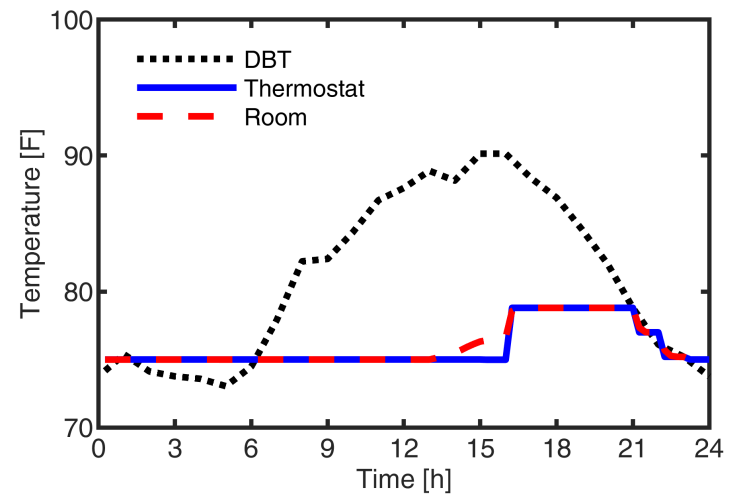

Figure 8. Dry bulb temperature (DBT), thermostat setting point, and room temperature for the equivalent house under HVAC control to reduce peak power. The thermostat setting point was changed from $75^{\circ} \mathrm{F}$ to $78^{\circ} \mathrm{F}$ between 16:00 and 21:00, and was then changed back to $75^{\circ} \mathrm{F}$.

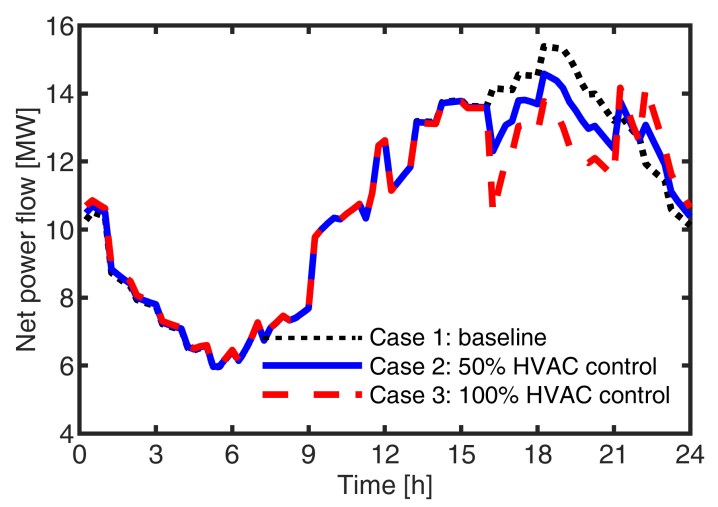

Figure 9. Simulation results for HVAC control to reduce peak power. The HVAC control was realized by changing the thermostat setting point. Peak power flow was reduced at around 18:00.

\section{REFERENCES}

[1] "EIA: consumption and effiency," https://https://www.eia.gov/ consumption/, accessed: 11/5/2019.

[2] "Residential Energy Survey: today in energy," https://www. eia.gov/todayinenergy/detail.php?id=36412\&src=\%E2\%80\%B9\% 20Consumption $\% 20 \% 20 \% 20 \% 20 \% 20 \% 20$ Residential $\% 20$ Energy $\%$ 20Consumption\%20Survey\%20(RECS)-b1, accessed: 11/5/2019.

[3] I. Beil, I. Hiskens, and S. Backhaus, "Frequency regulation from commercial building hvac demand response," Proceedings of the IEEE, vol. 104, no. 4, pp. 745-757, 2016.

[4] Q. Shi, C.-f. Chen, A. Mammoli, and F. Li, "Estimating the profile of incentive-based demand response (ibdr) by integrating technical models and social-behavioral factors," IEEE Transactions on Smart Grid, 2019.

[5] M. Olama, T. Kuruganti, J. Nutaro, and J. Dong, "Coordination and control of building hvac systems to provide frequency regulation to the electric grid," Energies, vol. 11, no. 7, p. 1852, 2018.

[6] H. Gong, V. Rallabandi, M. Michael L., and D. M. Ionel, "On the optimal energy controls for large scale residential communities including smart homes," in Proceedings, IEEE ECCE 2019, Baltimore, MD, $5 p$ (Oct 2019). IEEE, 2019, p. 5.

[7] O. Erdinc, A. Taşcıkaraoğlu, N. G. Paterakis, Y. Eren, and J. P. Catalão, "End-user comfort oriented day-ahead planning for responsive residential hvac demand aggregation considering weather forecasts," IEEE Transactions on Smart Grid, vol. 8, no. 1, pp. 362-372, 2016.

[8] Y. Yang, S. Li, W. Li, and M. Qu, "Power load probability density forecasting using gaussian process quantile regression," Applied Energy, vol. 213, pp. 499-509, 2018. 